\title{
A New Image Registration Technique With Free Boundary Constraints: Application To Mammography
}

\author{
F. Richard ${ }^{1}$ and L. Cohen ${ }^{2}$ \\ 1 MAP5, University Paris V René Descartes, 45 rue des Saints Pères, \\ 75270 Paris Cedex 06, France \\ 2 CEREMADE, University Paris IX Dauphine, \\ Place du Marechal de Lattre de Tassigny, 75775 Paris cedex 16, France \\ Coheneceremade.dauphine.fr
}

\begin{abstract}
In this paper, a new image-matching mathematical model is presented for the mammogram registration. In a variational framework, an energy minimization problem is formulated and a multigrid resolution algorithm is designed. The model focuses on the matching of regions of interest. It also combines several constraints which are both intensity and segmentation based. A new feature of our model is combining region matching and segmentation by formulation of the energy minimization problem with free boundary conditions. Moreover, the energy has a new registration constraint. The performances of models with and without free boundary are compared on a simulated mammogram pair. It is shown that the new model with free boundary is more robust to initialization inaccuracies than the one without. The interest of the new model for the real mammogram registration is also illustrated.
\end{abstract}

\section{Introduction.}

In this paper, we deal with the well-known problem of Image Registration. Our motivation is the registration of bilateral or temporal mammogram pairs. As can be seen though the complete survey in [9], a lot of work has been done in Image Registration since the early 80 's. However, the mammogram registration still remains a challenging problem. Indeed, most of the mammogram registration techniques have been only based on breast contours and for that reason have not succeeded in registering accurately breast interiors $[6,7,16,17]$. In [10,13-15], several authors attempted to register breast interiors using the Bookstein warping technique with internal control points [2]. However, these authors faced with the difficult problem of reliable internal breast control point extraction and matching.

In a recent work [11,12], F. Richard and C. Graffigne proposed an image-matching approach for the registration of mammogram pairs. This approach is not based on internal control points but instead on image grey-level values. It is more akin to the intensitybased approaches in $[1,3,8,19]$ than to the Bookstein technique. However, it differs significantly from these usual intensity-based approaches. On the one hand, it focuses on the mapping of regions of interest (the breast in the mammogram application) rather 
than the whole image matching. On the other hand, it combines some matching constraints which are intensity-based and segmentation-based. More precisely, it consists in minimizing an intensity-based energy with some boundary conditions (Dirichlet) which are derived from a preliminary breast contour match (see section 2.2).

In $[11,12]$, it was shown that thanks to these combined constraints the computation time and the mammogram registration accuracy are improved. However, the model performance depends on the quality of the preprocessing steps (the mammogram segmentations and the breast contour match). Indeed, since the boundary conditions are fixed, the preprocessing inaccuracies cannot be compensated for during the matching process. Hence these inaccuracies may decrease the matching performances. Besides, the Dirichlet boundary conditions constrain too strongly the problem and may sometimes disrupt the breast registration near the contours.

In this paper, we propose a model which fixes the drawbacks described above. As in $[11,12]$, the model enables the matching of regions of interest. But, contrarily to the previous model, the minimization problem is defined with free boundary conditions. Consequently, the boundary conditions are relaxed and it also becomes possible to compensate for the preprocessing inaccuracies during the matching process. Furthermore, some constraints are proposed in order to compensate efficiently for the preprocessing inaccuracies and increase the model robustness.

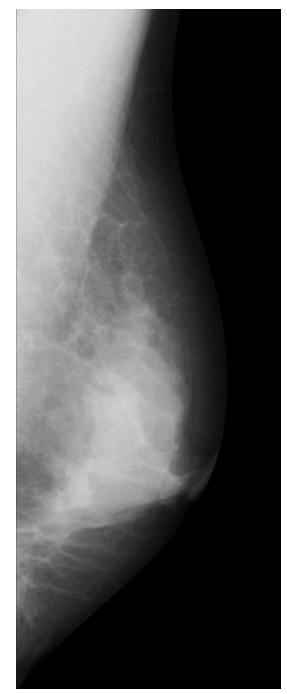

(a)

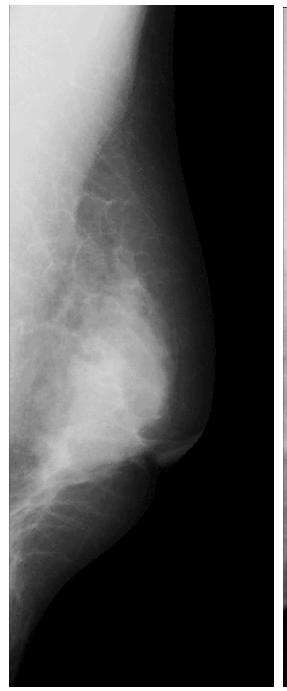

(b)

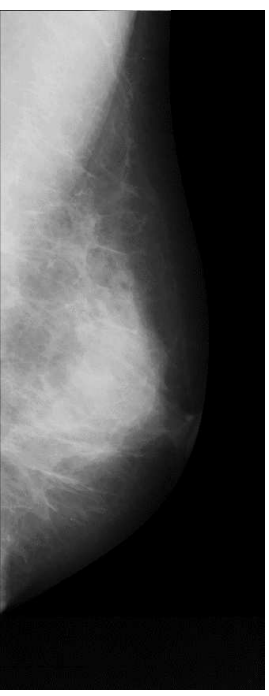

(c)

Fig. 1. (a) The source image $I^{0}$, (b) the geometric deformation $I_{\phi}^{0}$ of $I^{0}$ after the application of model 1 , (c) the target image $I^{1}$.

In section 2, the new image-matching approach is presented. In section 3, a multigrid algorithm is proposed for the numerical resolution of the problem. In section 4, it 
is given some preliminary illustrations and validations of the algorithm application to mammograms.

\section{Mathematical Problem Formulation.}

In this section, three different image-matching problems are formulated. In section 2.1, the formulation of the usual intensity-based problem is reminded. Sections 2.2 and 2.3 are both devoted to the matching of regions of interest. In section 2.2 , we recall the formulation of our previous model. In section 2.3, the new model is presented.

\subsection{The Classical Model.}

The classical variational framework for Image-Matching is the following $[1,3,8,19]$. Let $\Omega$ be a connected and open set of $\mathbb{R}^{2}$ and $I^{0}$ and $I^{1}$ be two images defined on $\Omega$ using interpolation. Let $\mathcal{W}_{a}$ be a space composed of smooth functions mapping $\bar{\Omega}$ onto itself. Let us denote by $I_{\phi}^{0}$ the geometric deformation of $I^{0}$ that is induced by the element $\phi$ of $\mathcal{W}_{a}$ :

$$
\forall x \in \Omega, I_{\phi}^{0}(x)=I^{0} \circ \phi(x) .
$$

Matching $I^{0}$ and $I^{1}$ consists in finding an element $\phi$ which is as smooth as possible and such that the deformed image $I_{\phi}^{0}$ is "similar" to $I^{1}$. This is expressed in terms of an inverse problem $[1,3,8,19]$ :

Model 1 Find in $\mathcal{W}_{a}$ a minimum for an energy $J_{a}$ which is of the following form:

$$
J_{a}(u)=\frac{1}{2} A_{\Omega}(u, u)+\frac{\gamma_{1}}{2}\left|I_{\phi}^{0}-I^{1}\right|_{\Omega}^{2},
$$

with some boundary conditions on the boundary of $\Omega$. In this energy definition, $u$ is equal to $\phi-\operatorname{Id}$ and $|\cdot|_{A}$ is the usual quadratic norm on $L^{2}(\bar{A} ; \mathbb{R}):|I|_{A}^{2}=\int_{A} I^{2}(x) d x$.

This energy is composed of two terms. The second term depends on the images. It is the matching term: the more similar the images $I_{\phi}^{0}$ and $I^{1}$ are, the lower this term is. The first term is a smoothing term which ensures that the problem has a solution in a space of homeomorphisms. Its design is usually based on a strain energy of the continuum media mechanics. In this paper, we choose the energy of the linearized elasticity [5]. Its definition can be based on following bilinear form:

$$
A_{\Omega}(u, v)=<L u, v>_{\Omega}=\int_{\Omega} L u(x) \cdot v(x) d x .
$$

Let us denote by $\langle\cdot, \cdot\rangle_{\Omega}$ the usual scalar product on $L^{2}\left(\bar{\Omega} ; \mathbb{R}^{2}\right)$ and by $L$ the following operator: ${ }^{1}$

$$
L u=-\operatorname{div}\{\lambda \operatorname{tr}(\mathbf{e}(u)) \operatorname{Id}+2 \mu \mathbf{e}(u)\} .
$$

where $\lambda$ and $\mu$ are two positive values and $\mathbf{e}(u)$ is the linearized strain tensor $1 / 2\left(\nabla u^{t}+\right.$ $\nabla u)$. In figure 1, it is shown an application of model 1 to a mammogram pair; this example will be commented further in section 4.

\footnotetext{
${ }^{1}$ If $M$ is a $2 \times 2$-matrix, then $\operatorname{tr}(M)$ is equal to $M_{1,1}+M_{2,2}$. If $m$ is a smooth function mapping $\Omega$ into the $2 \times 2$-matrix set, then the value of $\operatorname{div}\{m\}$ at a point $x$ of $\Omega$ is the bidimensional vector having the $i^{t h}$ component equal to $\partial_{x_{1}} m(x)_{i, 1}+\partial_{x_{2}} m(x)_{i, 2}$.
} 


\subsection{Region-Matching With Fixed Boundary Conditions.}

Unlike the previous model, the model presented in this section focuses on the matching of regions of interest. The framework is the following. Let us assume that images $I^{0}$ and $I^{1}$ have single regions of interest that are respectively located on the connected and open subsets $\Omega_{0}$ and $\Omega_{1}$ of $\Omega$. Let us denote by $\partial \Omega_{0}$ and $\partial \Omega_{1}$ the respective boundaries of $\Omega_{0}$ and $\Omega_{1}$. Let us assume that the contours $\partial \Omega_{0}$ and $\partial \Omega_{1}$ were previously segmented and matched. Let us denote by $\phi_{0}$ (or Id $+u_{0}$ ) the function mapping the coordinates of $\partial \Omega_{1}$ onto those of $\partial \Omega_{0}$. In order to focus on the regions of interest, the minimization problem is not defined on $\mathcal{W}_{a}$ (see section 2.1) but on a space $\mathcal{W}_{b}$ which is composed of smooth functions mapping $\overline{\Omega_{1}}$ onto $\overline{\Omega_{0}}$. The inverse problem is stated as follows [11, 12]:

Model 2 Find in $\mathcal{W}_{b}$ a minimum for an energy $J_{b}$ which is of the following form:

$$
J_{b}(u)=\frac{1}{2} A_{\Omega_{1}}(u, u)+\frac{\gamma_{1}}{2}\left|I_{\phi}^{0}-I^{1}\right|_{\Omega_{1}}^{2},
$$

with the following non-homogeneous Dirichlet boundary conditions:

$$
\forall x \in \partial \Omega_{1}, u(x)=u_{0}(x)=\phi_{0}(x)-x .
$$

The terms of energy $J_{b}$ have the same definitions and play the same roles as those of energy $J_{a}$ in model 1 . However, they are not defined on the whole domain $\Omega$ but only on the region of interest $\Omega_{1}$. Besides, the boundary conditions are specific to the regions of interest and based on a known matching of their contours. An application of model 2 is shown on figure 2 .

\subsection{Region-Matching With Free Boundary Conditions.}

The model presented in this section focuses on the regions of interest. But, unlike the previous model, the problem is defined with free boundary conditions. Hence, the problem is not defined on $\mathcal{W}_{b}$ (see section 2.2) but on a space $\mathcal{W}$ which is composed of smooth functions mapping $\overline{\Omega_{1}}$ onto $\mathbb{R}^{2}$. The inverse problem is defined as follows:

Find in $\mathcal{W}$ a minimum for an energy $J_{c}$ which is of the following form:

$$
J_{c}(u)=\frac{1}{2} A_{\Omega_{1}}(u, u)+\gamma_{1} \frac{1}{2}\left|I_{\phi}^{0}-I^{1}\right|_{\Omega_{1}}^{2}+\gamma_{2} \int_{\Omega-\phi\left(\Omega_{1}\right)} S\left(\left|I^{0}(x)\right|^{2}\right) d x,
$$

with free boundary conditions on $\partial \Omega_{1}$.

As in models 1 and 2, the energy has a matching and a regularity term. It has also a term which depends on the grey level values of $I^{0}$. This term is defined on a region $\Omega-\phi\left(\Omega_{1}\right)$ which is expected to be the background of $I^{0}$ (see figure 3 ). It constrains pixels of the set $\phi\left(\Omega_{1}\right)$ not to be located on the background of $I^{0}$. For reasons that will appear next, it will be refered as the segmentation term. An application of model 3 is shown on figure 2.

Design of $S$. Assume that the image $I^{0}$ can be robustly segmented using a threshold; that is to say there exists a value $\eta$ such that $\left|I^{0}(x)\right|^{2}<\eta$ if and only if $x$ belongs to the 


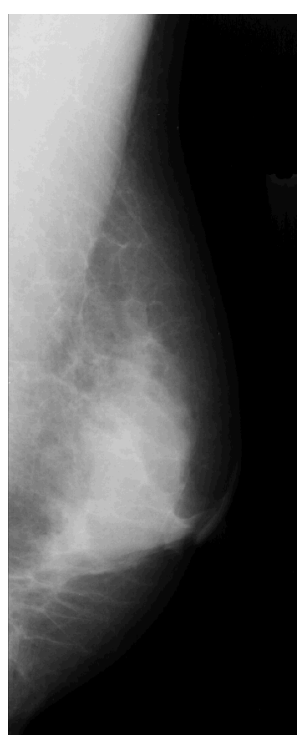

(a)

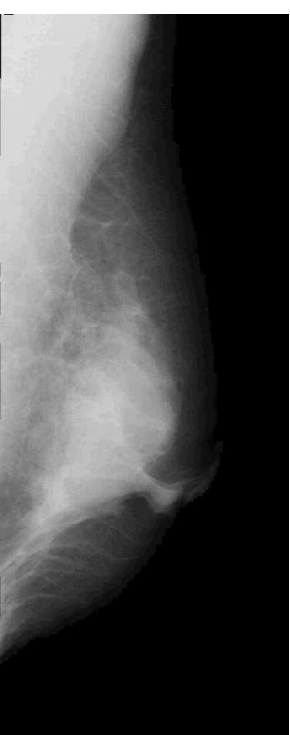

(b)

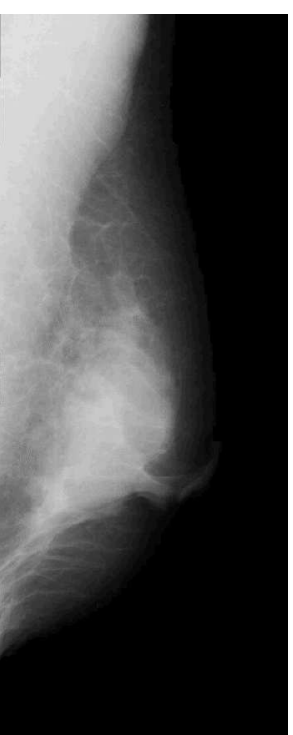

(c)

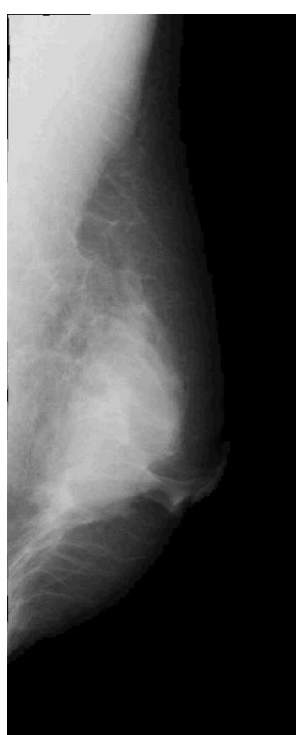

(d)

Fig. 2. (a) The source image $I^{0}$, the geometric deformation $I_{\phi}^{0}$ of $I^{0}$ after the application of (b) model 2 and (c) model 3, (d) the target image $I^{1}$.

background of the image $I^{0}$. Then, $S$ can be defined as a smooth distribution function approximating on a bounded interval the function that is equal to 0 on ] $-\infty, \eta$ [ and 1 on $[\eta,+\infty$. The value of $S$ at point $r$ of $\mathbb{R}$ may be interpreted as the conditional probability for a pixel $x$ not to be on the image background knowing that $\left|I^{0}(x)\right|^{2}$ is equal to $r$. In the case where the segmentation is not reliable, the design of $S$ can be based on an empirical estimation of these probabilities. In the mammogram application, these probabilities are estimated using image $I^{1}$ for which segmentation is known. The value of $S$ at $r$ is defined as the frequency of pixels of $\Omega_{1}$ for which $\left|I^{1}(x)\right|^{2}$ is equal to $r$.

Segmentation of $I^{0}$. Contrary to model 2, a preliminary segmentation of the region of interest in $I^{0}$ is not needed for the problem formulation. A segmentation of $I^{0}$ may be obtained after the problem resolution: the contour is given by the image $\phi\left(\partial \Omega_{1}\right)$ of $\partial \Omega_{1}$ by a function $\phi$ which minimizes the energy.

An equivalent problem. Assuming some regularity conditions (the elements $\phi$ of $\mathcal{W}$ belong to the Sobolev space $W^{1,2}\left(\overline{\Omega_{1}} ; \mathbb{R}^{2}\right)[5]$ and are such that $\operatorname{det}(\nabla \phi)>0$ on $\Omega_{1}$ ), it can be seen that

$$
\int_{\Omega-\phi\left(\Omega_{1}\right)} S\left(\left|I^{0}(x)\right|^{2}\right) d x=\int_{\Omega} S\left(\left|I^{0}(x)\right|^{2}\right) d x-\int_{\Omega_{1}} S\left(\left|I_{\phi}^{0}(x)\right|^{2}\right) \operatorname{det}(\nabla \phi) d x .
$$

Thus, the previous minimization problem can be expressed in the equivalent way: 
Model 3 Find in $\mathcal{W}$ a minimum for an energy $J$ which is of the following form:

$$
J(u)=\frac{1}{2} A_{\Omega_{1}}(u, u)+\frac{\gamma_{1}}{2}\left|I_{\phi}^{0}-I^{1}\right|_{\Omega_{1}}^{2}-\gamma_{2} \int_{\Omega_{1}} S\left(\left|I_{\phi}^{0}(x)\right|^{2}\right) \operatorname{det}(\nabla \phi) d x,
$$

with free boundary conditions on $\partial \Omega_{1}$.

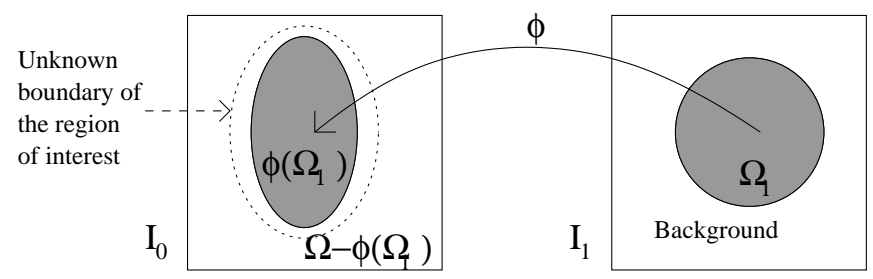

Fig. 3. A schematic picture of region-matching with free boundary conditions.

\section{Problem Resolution.}

In this section, a gradient descent algorithm is designed for the numerical resolution of the problem of model 3. In section 3.1, the energy is derived and the algorithm is expressed in terms of an ODE (Ordinary Differential Equation). In section 3.2, we propose a spatial discretization of the ODE using the Galerkin method. In section 3.3, an ODE initialization method is described. In section 3.4, a multigrid implementation of the algorithm is designed.

\subsection{Gradient Descent Algorithm.}

The Frechet derivative of the energy $J$ (equation (3)) at point $u$ of $\mathcal{W}$ is as follows: for all $v$ in $\mathcal{W}$

$$
\begin{aligned}
& d J_{\left.\right|_{u}}(v)=A_{\Omega_{1}}(u, v)+\gamma_{1}<\left(I_{\phi}^{0}-I^{1}\right) \nabla I_{\phi}^{0}, v> \\
& -2 \gamma_{2} \int_{\Omega_{1}} \operatorname{det}(\nabla \phi) S^{\prime}\left(\left|I_{\phi}^{0}\right|^{2}\right) \nabla I_{\phi}^{0} \cdot v d x-\gamma_{2} \int_{\Omega_{1}} S\left(\left|I_{\phi}^{0}\right|^{2}\right) \operatorname{tr}\left(\operatorname{cof}(\nabla \phi)^{t} \cdot \nabla v\right) d x
\end{aligned}
$$

where $\operatorname{cof}(M)$ denotes the cofactor matrix of a matrix $M$. But, by a Green formula [5],

$$
\int_{\Omega_{1}} S\left(\left|I_{\phi}^{0}\right|^{2}\right) \operatorname{tr}\left(\operatorname{cof}(\nabla \phi)^{t} \cdot \nabla v\right) d x=-\int_{\Omega_{1}} \operatorname{div}\left\{S\left(\left|I_{\phi}^{0}\right|^{2}\right) \operatorname{cof}(\nabla \phi)\right\} \cdot v d x
$$

the gradient of the energy $J$ with respect to the inner product $A_{\Omega_{1}}(\cdot, \cdot)$ is given by:

$$
\nabla J_{u}=u-L^{-1} F(\phi(t)),
$$


where $L$ is the operator defined by equation (2) and $F$ is the following mapping:

$$
\begin{aligned}
& F(\phi)=-\gamma_{1}\left(I_{\phi}^{0}-I^{1}\right) \nabla I_{\phi}^{0} \\
& \quad+2 \gamma_{2} \operatorname{det}(\nabla \phi) S^{\prime}\left(\left|I_{\phi}^{0}\right|^{2}\right) \nabla I_{\phi}^{0}-\gamma_{2} \operatorname{div}\left\{S\left(\left|I_{\phi}^{0}\right|^{2}\right) \operatorname{cof}(\nabla \phi)^{t}\right\}
\end{aligned}
$$

Thus, the gradient descent of the energy $J$ can be expressed in terms of the following ODE:

\section{Algorithm 1 (gradient descent)}

$$
\forall t>0, \frac{d u}{d t}(t)=-u(t)+\delta(t) \text { and } u(0)=M_{0},
$$

where the initial deformation $M_{0}$ will be defined in section 3.3, and at each time $t \delta(t)$ is the solution of the following PDE (Partial Derivative Equation):

$$
L \delta=F(\phi(t)) \text {, }
$$

where $\phi(t)=\operatorname{Id}+u(t)$ and $F$ is defined in equation (5).

\subsection{Algorithm Discretization.}

For the implementation of algorithm 1, equation (7) is discretized using the Galerkin method [4]. First, it can be noticed that equation (7) is formally equivalent to the variational equation:

$$
\forall v \in \mathcal{W}, A_{\Omega_{1}}(\delta, v)=\langle F(\phi(t)), v>
$$

where $F$ is defined in equation (5). We choose a space $\mathcal{W}^{h}$ of dimension $h$ which is included in $\mathcal{W}$ and spanned by a finite family of functions with compact support (denoted by $\left\{\psi_{i}^{h}\right\}_{i \in I_{h}}$ ). In order to approximate the solution of equation (8), we find in $\mathcal{W}^{h}$ the solution of the approximate variational equation:

$$
\forall v \in \mathcal{W}^{h}, A_{\Omega_{1}}(\delta, v)=\langle F(\phi(t)), v>.
$$

The solution of this equation is the following:

$$
\delta^{h}=\sum_{j \in I_{h}} \beta_{j}^{h} \psi_{j}^{h},
$$

where the coefficients $\beta_{j}^{h}$ are the solution of the linear system:

$$
\forall i \in I_{h}, \quad \sum_{j \in I_{h}} \beta_{j} A_{\Omega_{1}}\left(\psi_{j}^{h}, \psi_{i}^{h}\right)=<F(\phi(t)), \psi_{i}^{h}>
$$

In order to design the approximation spaces $\mathcal{W}^{h}$, the set $\Omega_{1}$ is decomposed into $h / 2$ fixed-size non-overlapping squares. We define $\mathcal{W}^{h}$ as the space formed by the maps that are $\mathcal{C}^{1}$ on $\Omega_{1}$ and polynomial on each of these squares. The design of the function family $\left\{\psi_{i}^{h}\right\}_{i \in I_{h}}$ is based on spline functions.

When decomposed, the domain $\Omega_{1}$ may be slightly approximated near the boundaries. This may cause segmentation inaccuracies. However, these inaccuracies are taken into account in model 3 via the estimation of $S$ (see section 2.3). 


\subsection{Initialization Step.}

Unlike model 2, the contour match is not used for the design of model 3. However, it is worth using it to have a better initialization of the ODE. Hence we define the displacements $M_{0}$ in equation (6) as the solution of the problem in model 2 when $\gamma_{1}$ is equal to zero. The displacements $M_{0}$ are the same as those which are obtained at the initialization step of the algorithm of model $2[11,12]$. Let us denote by $\mathcal{W}_{0}$ the space composed of the functions of $\mathcal{W}_{b}$ (see section 2.2) and equal to 0 on $\partial \Omega_{1}$. The displacements $M_{0}$ are equal to $u_{0}+\delta_{0}$, where $u_{0}$ is defined in section 2.2 and $\delta_{0}$ is the solution in $\mathcal{W}_{0}$ of the following variational equation:

$\forall v \in \mathcal{W}_{0}, A_{\Omega_{1}}(\delta, v)=-A_{\Omega_{1}}\left(u_{0}, v\right)$. Using the Galerkin method (see section 3.2), $\delta_{0}$ can be approximated by the displacements $\delta_{0}^{h}$ which are found as follows:

$$
\delta_{0}^{h}=\sum_{j \in I_{h}} \beta_{j, 0}^{h} \psi_{j}^{h} \in \mathcal{W}_{0}^{h},
$$

where the coefficients $\beta_{j, 0}^{h}$ are the solution of the linear system

$$
\forall i \in I_{h}, \sum_{j \in I_{h}} \beta_{j, 0}^{h} A_{\Omega_{1}}\left(\psi_{j}^{h}, \psi_{i}^{h}\right)=-A_{\Omega_{1}}\left(u_{0}, \psi_{i}^{h}\right) .
$$

\subsection{Multigrid Implementation.}

In order to lower computation times and obtain better minimization results, we adopt a multigrid implementation approach together with a coarse-to-fine strategy. We define a series $\left\{\mathcal{W}^{h(k)}\right\}_{k \in \mathbb{N}}$ of embedded subspaces having the properties described in section 3.2:

$$
\mathcal{W}^{h(1)} \subset \cdots \subset \mathcal{W}^{h(k)} \subset \cdots \subset \mathcal{W} .
$$

The ODE is descritized with respect to time using the Euler method. We obtain the following resolution scheme:

\section{Algorithm 2 (Multigrid Implementation)}

Initialization: $u(0)=u_{0}+\delta_{0}^{h(K)}$, where $u_{0}$ is defined in section 2.2 and $\delta_{0}^{h(K)}$ is the solution in a space $\mathcal{W}_{0}^{h(K)}$ of equations (11) and (12).

$\mathbf{k}^{\text {th }}$ Iteration $(k \geq 0): u(k+1)=u(k)+\epsilon \delta(k)$, where $\epsilon$ is a small positive value and $\delta(k)$ is the solution in $\mathcal{W}^{h(k)}$ of equations (9) and (10) with tequal to $k$.

\section{Application to mammogram pairs.}

The local comparison of bilateral or temporal mammogram pairs is an usual approach for the automatic detection of abnormalities $[6,7,16]$. For this approach to be efficient, the mammogram couples have to be registered. Indeed, unregistered mammograms have a lot of normal differences which can be taken as abnormalities and generate high false positive rates. At the finest scale, mammograms are too different to be matched by 
homeomorphisms. Thus, a reasonable registration goal is to remove the image differences which are present at a coarse scale (differences of contours and of bright and dark regions). In what follows, we only register coarse approximations of mammograms.

In this section, we apply the different models described in section 2 to mammogram pairs. In section 4.1 , it is given some preliminary evaluations and comparisons of the algorithm performances based on a simulated mammogram pair. In section 4.2 , we illustrate the algorithm applications to real mammogram pairs.

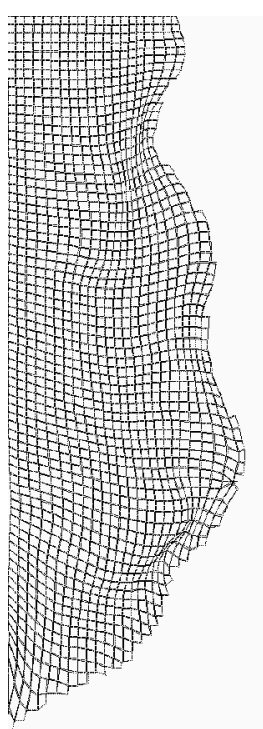

(a)

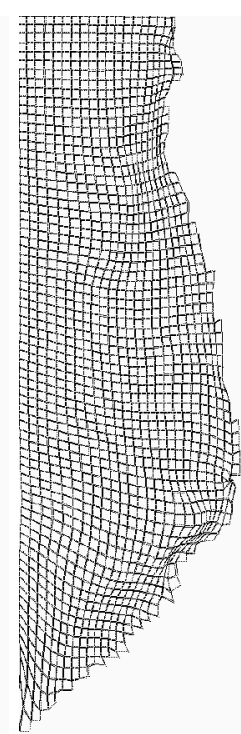

(b)

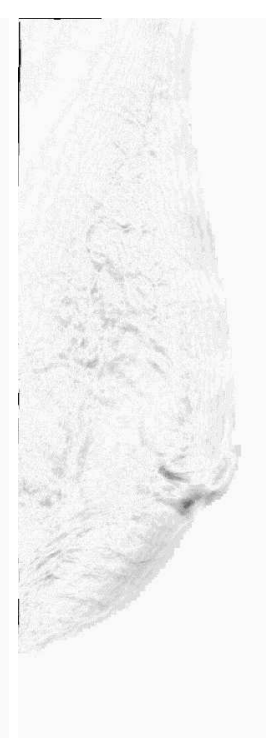

(c)

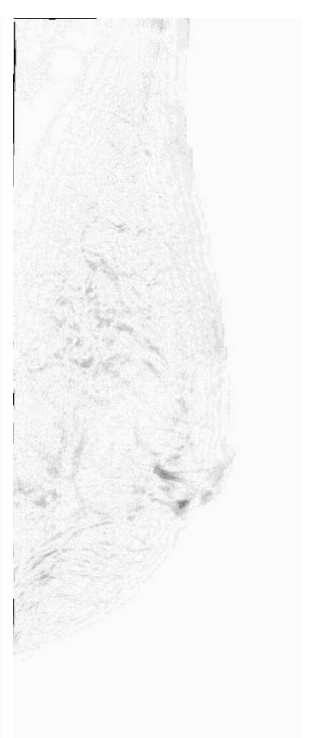

(d)

Fig. 4. Figures (a) and (b) shows the image $\phi\left(\Omega_{1}\right)$ of the tessellated breast domain $\Omega_{1}$ in $I^{1}$ by the solution $\phi$ obtained with the algorithm of model 2 (figure (a)) and model $3\left(\gamma_{2}=1000\right.$ ) (figure (b)). Figures (c) and (d) shows the absolute differences between images $I^{1}$ and $I^{0} \circ \phi$ where $\phi$ are the solutions found using the algorithm of model 3 with $\gamma_{2}=0$ (figure (c)) and with $\gamma_{2}=1000$ (figure (d)). [black=high differences, white=low differences]

\subsection{A simulated case.}

In figures 2 (a) and (c), it is shown a simulated mammogram pair $\left(I^{0}, I^{1}\right)$ where $I^{1}$ is a geometric deformation of $I^{0}: I^{1}=I^{0} \circ \phi^{\star}$. The function $\phi^{\star}$ is known. It results from the application of model 2 to the real mammogram pair $\left(I^{0}, I_{\star}^{1}\right) ; I_{\star}^{1}$ is shown on figure 6 (f). The algorithm of model 2 was applied to the simulated image pair with the exact initialization derived from $\phi^{\star}$. A solution denoted by $\phi_{\text {ref }}$ was obtained. As can be seen on figure 2 (c), the images are almost perfectly registered by this algorithm. The image difference was lowered by 80.8 percent and the mean distance between the algorithm 
solution $\phi_{\text {ref }}$ and the exact mapping $\phi^{\star}$ is 3.8 pixels. The image registration benefits from the region-specific constraints: the algorithm of model 1 lowered the image difference by only 68 percent. In this case, model 2 is the most relevant among the three models described above since the boundary conditions are exact.

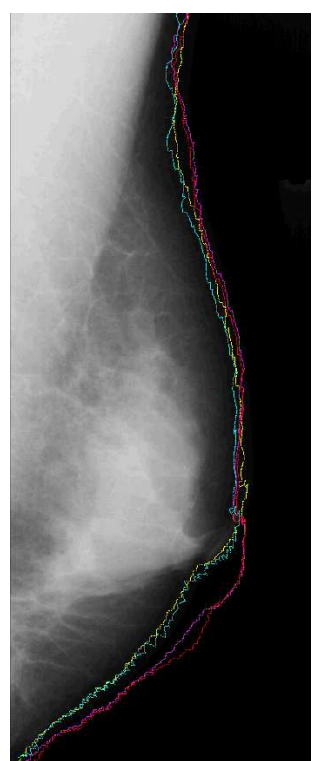

(a)

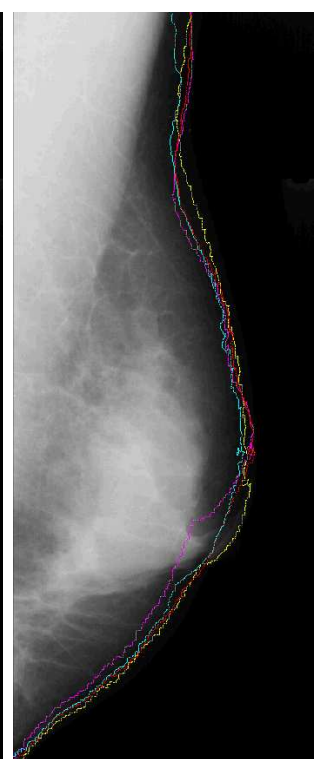

(b)

Fig. 5. Correction effect of the segmentation term of model 3. In figures (a) and (b), the yellow line is the correct segmentation of the breast and the pink one is the segmentation which is induced by the wrong initialization map $\phi_{0}$. The blue and red lines are the segmentations that are induced by the solutions of the algorithm of model 3 with $\gamma_{2}$ equal to 0 and 1000 respectively.

Based on $\phi_{\star}$, we designed five wrong initialization functions $\phi_{0}$ defined on the breast contour of $I^{1}$ (see sections 2.2 and 3.3). Comparing the yellow and pink lines in figures 5 (a) and (b), it can be seen that these functions do not correctly map into the breast contour in $I^{0}$. The algorithms of models 2 and 3 were applied to $I^{0}$ and $I^{1}$ with the wrong initializations functions. The mean results are shown in table 1 . It can be observed that the registration performance of model 2 is drastically reduced due to the initialization errors. Indeed, the image difference is lowered by only 69.9 percent on average with wrong initializations. Moreover, the solution regularity is decreased: the regularity term reaches the mean value 344.5 with wrong initializations whereas it was only 117 with the exact initialization. This regularity decrease is due to some compressions which can be observed in figure 4 (a) near the breast contours. These compressions are caused by the opposition of the two registration contraints (the fixed and wrong boundary conditions and the intensity-based energy term). 


\begin{tabular}{|c|c|c|c|c|c|c|c|c|c|c|c|c|}
\hline & \multirow{2}{*}{\multicolumn{2}{|c|}{$\begin{array}{c}\text { Initialization } \\
\text { Step }\end{array}$}} & \multirow{2}{*}{\multicolumn{2}{|c|}{$\begin{array}{l}\text { Algorithm } \\
\text { of Model } 2\end{array}$}} & \multicolumn{8}{|c|}{ Algorithm of Model 3} \\
\hline & & & & & $\gamma_{2}$ & $=0$ & $\gamma_{2}=$ & 500 & $\gamma_{2}=$ & 1000 & $\gamma_{2}=$ & 10000 \\
\hline & Regis. & Regul. & Regis. & Regul. & Regis. & Regul & Regis. & $\overline{\text { Regul. }}$ & Regis & Regul. & Regis & Regul. \\
\hline Mean & 19.7 & 295.5 & 69.9 & 344.2 & 74.1 & 301 & 75.5 & 298.6 & 76.9 & 300 & 76.1 & 313.8 \\
\hline Std Dev & 18.4 & 124.1 & 2.4 & 139.2 & 3.9 & 129.8 & 3.2 & 130.0 & 1.8 & 129.1 & 1.1 & 128.2 \\
\hline
\end{tabular}

Table 1. Comparison of the applications of models 2 and 3 with wrong initializations. The columns "Regis." give the image registration scores (in percentage of the initial quadratic difference between $I^{0}$ and $\left.I^{1}\right): 100 \cdot\left(\left|I^{0}-I^{1}\right|^{2}-\left|I_{\phi}^{0}-I^{1}\right|^{2}\right) /\left|I^{0}-I^{1}\right|^{2}$. The columns "Regul." give the values of the regularity term (equation (1)). The row "Mean" gives the means of the registration and regularity scores of the algorithms with five different wrong initializations and the row "Std Dev" the standard deviations of these scores.

Model 3 is more robust than model 2 to the initialization errors. Indeed, the registration score is higher (more than 74.1 percent in mean) and the solution are smoother. Comparing 4 (a) and (b), it can also observed that the compressions near the contours are less important in the solution of model 3 than in that of model 2 . The robustness of model 3 is further attested by the comparisons of the algorithm solutions and the reference solution $\phi_{\mathrm{ref}}$ : the mean and the standard deviation of the distances between the solutions obtained with model 2 and $\phi_{\text {ref }}$ are 9.6 and 7.1 pixels respectively whereas they are only 7.1 and 3.2 between the solutions of model $3\left(\gamma_{2}=0\right)$ and $\phi_{\text {ref }}$ and 6.1 and 3.1 between the solutions of model $3\left(\gamma_{2}=1000\right)$ and $\phi_{\text {ref. }}$. Besides, it can be noticed that the performance of model 3 improves as the weight $\gamma_{2}$ of the segmentation term increases. When $\gamma_{2}$ is equal to 1000 , the algorithm of model 3 not only obtains the registration score that is the closest to that of the solution $\phi_{\text {ref }}$ but also the results with the lowest standard deviations. This shows that the segmentation term in model 3 is a factor of robustness.

Comparing the image differences in figures 4 (c) and (d), it can be observed that the mammograms are much better registered near the contours when $\gamma_{2}$ is high. In figures 5 (a) and (b), it is shown the segmentations of $I^{0}$ which are induced by the algorithm solutions. It can be seen that the segmentation obtained with model 3 when $\gamma_{2}$ is high is close to the right segmentation whereas the segmentation obtained with model 3 when $\gamma_{2}$ is low remains close to the wrong initialization segmentation. In model 3, the segmentation term is necessary for the initialization errors to be compensated for.

\subsection{Application to a real mammogram pair.}

The bilateral mammogram pair shown in figures 6 (a) and (f) comes from the MIAS database [18]. Comparing both images and observing their absolute differences (figure 7 (a)), it can be noticed that they have a lot of important asymmetries, due in particular to differences in breast shape. Next, looking at the image $I^{0}$ in figure 6 (a) and the geometric deformation $I_{\phi}^{0}$ in figure 6 (b), it can be seen that the initialization step changes the breast shape and the nipple position in $I^{0}$. As observed on figure 7 (b), these changes significantly compensate not only for the asymmetries near the breast contours but also for some inner differences. However, due to preprocessing step inaccuracies and to the algorithm discretization (see section 3.2), the breast contour asymmetries are 


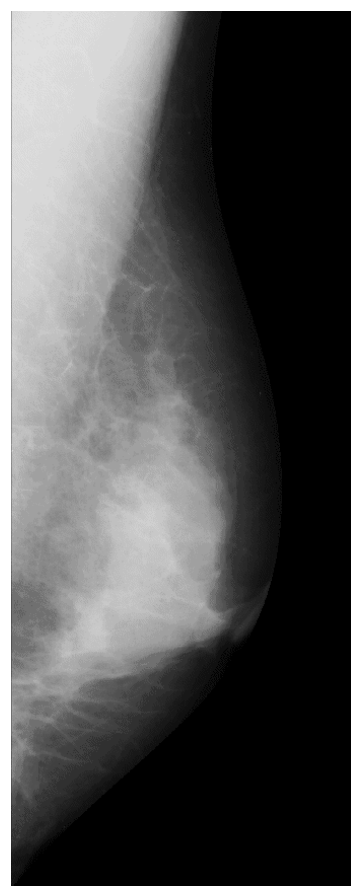

(a)

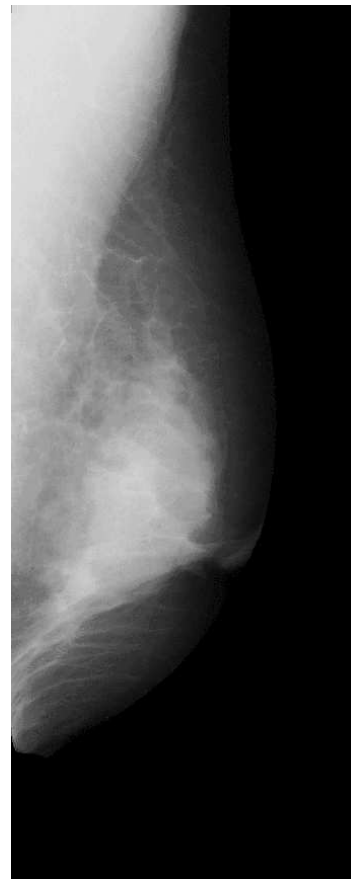

(d)

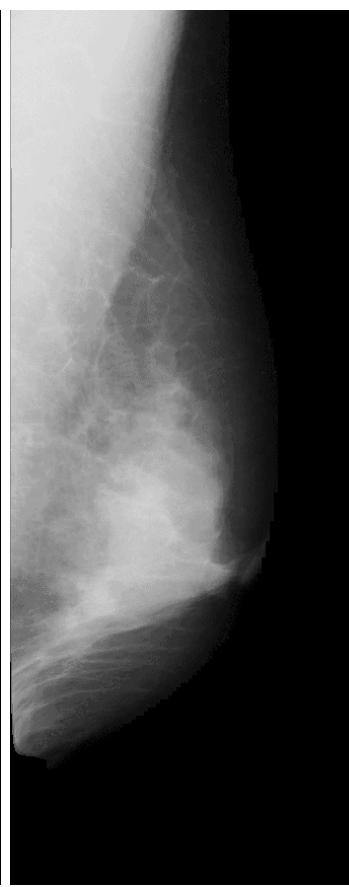

(b)

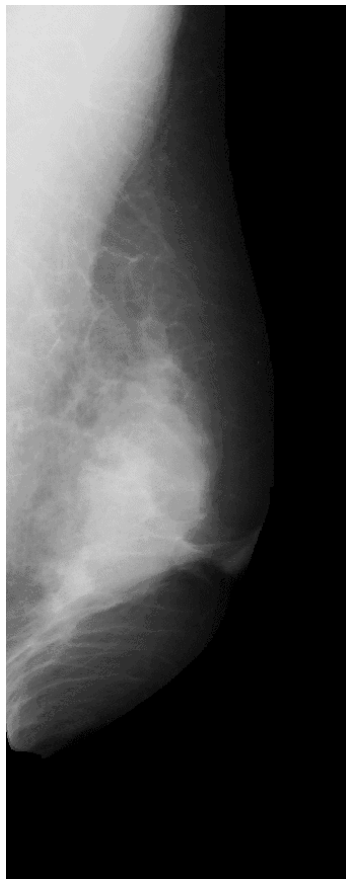

(e)

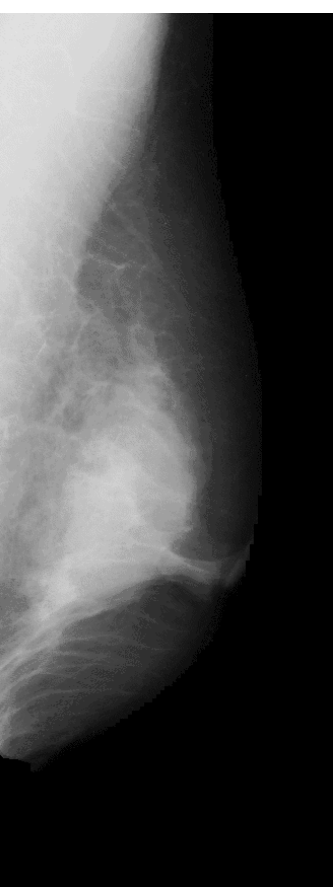

(c)

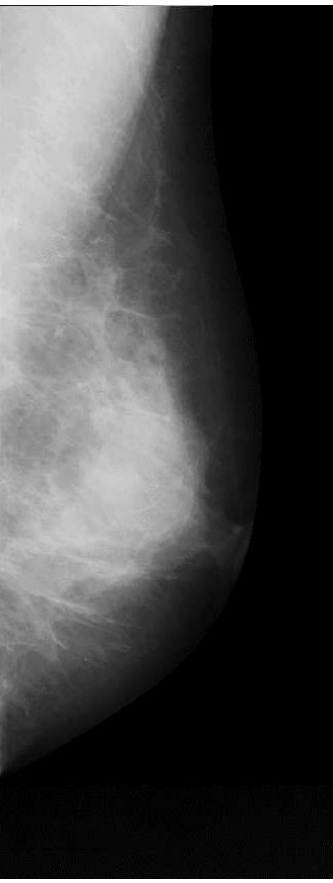

(f)

Fig. 6. (a) The source image $I^{0}$, the geometric deformation $I_{\phi}^{0}$ of $I^{0}$ (b) after the initialization step, (c) after the application of model 2, (d) after the application of model 3 with $\gamma_{2}$ equal to 0 , (e) after the application of model 3 with $\gamma_{2}$ equal to 1000 , (f) the target image $I^{1}$. 


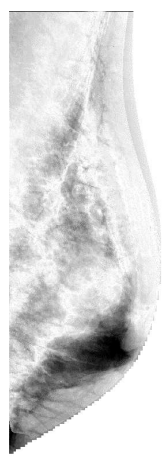

(a)

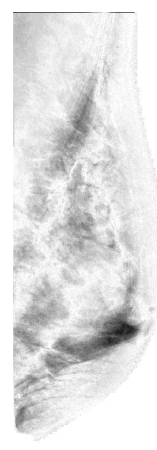

(b)

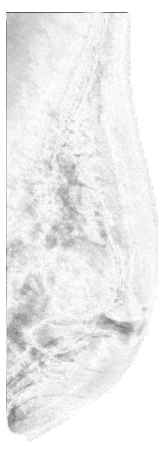

(c)

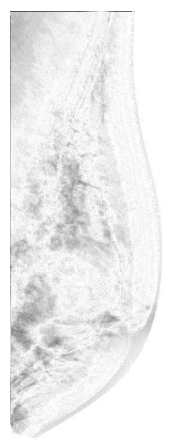

(d)

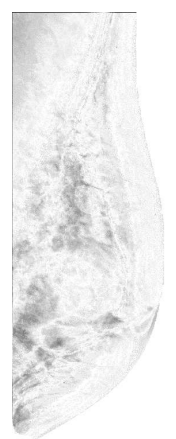

(e)

Fig. 7. The absolute differences between image $I^{1}$ and (a) $I^{0}$, (b) the deformed image $I_{\phi}^{0}$ after the initialization step, (c) the deformed image $I_{\phi}^{0}$ after the application of model 2, (d) the deformed image $I_{\phi}^{0}$ after the application of model 3 with $\gamma_{2}$ equal to 0 , (e) the deformed image $I_{\phi}^{0}$ after the application of model 3 with $\gamma_{2}$ equal to 1000 [black=high differences, white=low differences]

not perfectly corrected: in figure 6 (b), it can be observed a small dark border in the upper part of the contour area. Moreover, several important inner differences remain in the corrected image pair.

However, most of these inner differences can be removed by applying the algorithm of model 2. Indeed, comparing the images $I_{\phi}^{0}$ in figures 6 (c) and figure 6 (f) and looking at figure 7 (c), it can be seen that this algorithm compensates for many important asymmetries which are due to the shape and location differences of the bright regions in both images. Looking at figures 1 (e) and 7 (c), it can also be noticed that model 2 gives a better registration than model 1, particularly near the contours. However, several differences still remain in the pair of images corrected by model 2 . Since they are caused by breast tissue disparities, some of these differences cannot be corrected by any geometric deformation (see for instance, the tissue brightness disparity in the bottom left part of the images).

However, this registration can be improved. In particular, in the corrected image pair (figure 7 (c)), we still observe the contour differences which remained after the initialization. These differences cannot be compensated for by model 2 due to the fixed boundary conditions. Besides, an important difference remains near the nipple position. The algorithm of model 2 is unable to correct it: the difference increases the intensitybased matching term constraint in a way that is opposite to the boundary constraints. As can be noticed in figure 8 (b), the conflict of constraints generates a strong compression in the difference area. Further difference corrections are not possible because they would increase the compression and the regularity term value (equation (1)).

These results are in sharp contrast with those of model 3. We recall that, in this model, the boundary conditions are free. Consequently, the contour constraint of model 2 is less stringent. This relaxation permits a better registration of the images near the 


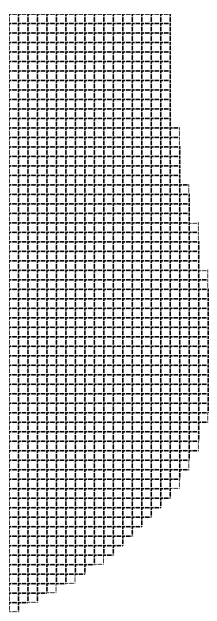

(a)

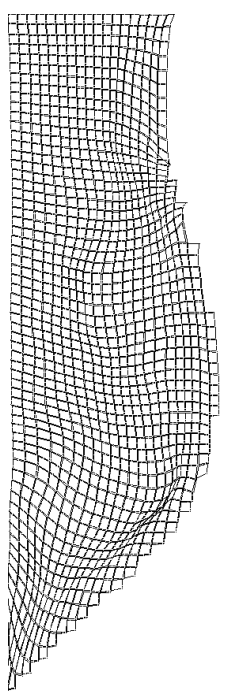

(b)

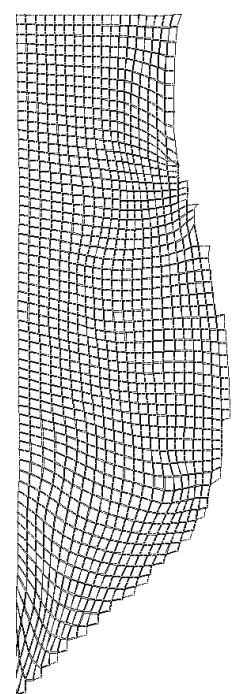

(c)

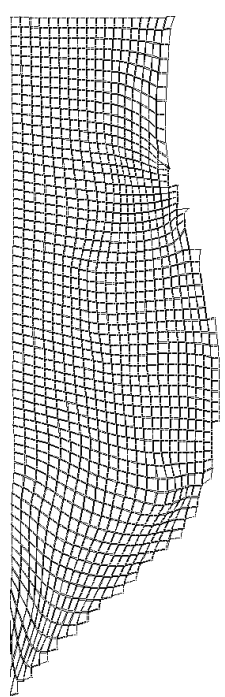

(d)

Fig. 8. (a) The tessellated breast domain $\Omega_{1}$ in $I^{1}$, the image $\phi\left(\Omega_{1}\right)$ of $\Omega_{1}$ by the map $\phi$ obtained with (b) the application of model 2, (c) the application of model 3 with $\gamma_{2}$ equal to 0 , (d) the application of model 3 with $\gamma_{2}$ equal to 1000 .

breast contours. Looking at figures 6 (d) and (e) and figures 7 (d) and (e), it can be observed that the algorithm (with different segmentation term weights $\gamma_{2}$ ) succeeds in compensating completely for the differences near the nipples. Moreover, as can be observed in figures 8 (c) and (d), the deformations are less compressed and smoother than the ones obtained with model 2. As can be observed on figures 7 (d) and (e), the algorithm maps without the segmentation term $\left(\gamma_{2}=0\right)$ some parts of the breast in $I^{1}$ into some parts of the background in $I^{0}$ whereas the algorithm with a strong segmentation constraint keeps the mapping inside the breast region of $I^{0}$.

\section{Conclusion.}

It was presented a new variational model for the mammogram registration. An energy minimization problem was formulated. A multigrid gradient descent algorithm was designed for the numerical resolution of the problem. As in [11, 12], the model focuses on the matching of regions of interest. It also combines segmentation-based and intensitybased constraints. However, the energy minimization problem is not posed with fixed boundary conditions but with free boundary conditions. Moreover, the energy has a new registration constraint. The performances of both models were compared on a simulated mammogram pair. It was shown that the new model is more robust to the initialization inaccuracies than the previous one. It was also illustrated the ability of the new model to compensate for these inaccuracies during the matching process. Both models were 
applied to a real mammogram pair in order to illustrate the interest of the new model in this application context. Although it was designed for the mammogram registration, the model is generic: it can be applied whenever the images have single regions of interest. We believe that, in these common cases, the new model is better suited for image registration than the usual intensity-based models.

\section{References}

1. R. Bajcsy and S. Kovacic. Multiresolution elastic matching. CVGIP, 46:1-21, 1989.

2. F.L. Bookstein. Principal warps: thin-plate splines and the decomposition of deformations. IEEE PAMI, 11(6):567-585, 1989.

3. G.E. Christensen, R.D. Rabbitt, and M.I. Miller. Mathematical textbook of deformable neuroanatomies. In Proc. Natl. Acad. Sci., USA, volume 90, pages 11944-11948, dec 93.

4. P. Ciarlet. The finite element method for elliptic problems. North-Holland, Amsterdam, 1978.

5. P. Ciarlet. Mathematical elasticity, volume 1: three-dimensional elasticity. Studies in Mathematics and its Applications. North-Holland, Amsterdam, 1988.

6. N. Karssemeijer and G.M. te Brake. Combining single view features and asymmetry for detection of mass lesions. In N. Karssemeijer et al., editors, Proc. IWDM, pages 95-102, Nijmegen, The Netherlands, June 1998. Kluwer Academic.

7. T.K. Lau and W.F. Bischof. Automated detection of breast tumors using the asymmetry approach. Comput. Biomed. Res., 24(3):273-295, 1991.

8. M. Lefébure and L. Cohen. Image registration, optical flow and local rigidity. JMIV, 14(2), mar 2001.

9. J. Maintz and M.A. Viergever. A survey of medical image registration. Medical Image Analysis, 2(1):1-36, 1998.

10. K. Marias and J.M. Brady. Multi-scale landmark selection for improved registration of temporal mammograms. In M. Yaffe, editor, Proc. IWDM, pages 580-586, Toronto, Canada, June 2000. Medical Physics Publishing.

11. F. Richard. Modèles élastiques d'ajustements d'images et applications aux paires bilatérales et temporelles de mammographies. PhD thesis, Université Paris V, France, dec 2000.

12. F. Richard and C. Graffigne. An image-matching model for the registration of temporal or bilateral mammogram pairs. In M. Yaffe, editor, IWDM, pages 756-762, Toronto, Canada, June 2000. Medical Physics.

13. M. Sallam and K. Bowyer. Detecting abnormal densities in mammograms by comparison to previous screenings. In K. Doi et al., editors, Proc. IWDM, pages 417-420, Chicago, USA, June 1996. Elsevier Science.

14. M.Y. Sallam and K. Bowyer. Registering time sequences of mammograms using a twodimensional image unwarping technique. In A.G. Gale et al., editors, Proc. IWDM, pages 121-130, York, England, July 1994. Elsevier Science.

15. M.Y. Sallam and K. Bowyer. Registration and difference analysis of corresponding mammogram images. Medical Image Analysis, 3(2):103-118, 1999.

16. J.L. Semmlow, A. Shadagopappan, L.V. Ackerman, et al. A fully automated system for screening Xeromammograms. Comput. Biomed. Res., 13:350-362, 1980.

17. A. Stamatakis, A.Y. Cairns, I.W. Ricketts, et al. A novel approach to aligning mammograms. In A. Gale et al., editors, Proc. IWDM, pages 355-364, England, jul 1994. Elsevier Science.

18. J. Suckling, J. Parker, D.R. Dance, et al. The Mammographic Image Analysis Society digital mammogram database. In A.G. Gale et al., editors, Proc. IWDM, York, England, July 1994.

19. A. Witkin, T. Terzopoulos, and M. Kass. Signal matching through scale space. IJCV, 1(2):133-144, 1987. 